$11-1-2013$

\title{
Intrinsically Ties Adjusted Non-Parametric Method for the Analysis of Two Sampled Data
}

\author{
G. U.Ebuh \\ Nnamdi Azikiwe University, Awka, Nigeria, ablegod007@yahoo.com
}

I. C. A Oyeka

Follow this and additional works at: http://digitalcommons.wayne.edu/jmasm

Part of the Applied Statistics Commons, Social and Behavioral Sciences Commons, and the Statistical Theory Commons

\section{Recommended Citation}

Ebuh, G. U. and Oyeka, I. C. A (2013) "Intrinsically Ties Adjusted Non-Parametric Method for the Analysis of Two Sampled Data," Journal of Modern Applied Statistical Methods: Vol. 12 : Iss. 2 , Article 8.

DOI: $10.22237 /$ jmasm/1383278820

Available at: http://digitalcommons.wayne.edu/jmasm/vol12/iss2/8 


\section{Intrinsically Ties Adjusted Non-Parametric Method for the Analysis of Two Sampled Data}

\author{
G. U. Ebuh \\ Nnamdi Azikiwe University \\ Awka, Nigeria
}

\author{
I. C. A. Oyeka \\ Nnamdi Azikiwe University \\ Awka, Nigeria
}

A non-parametric method for the analysis of two sample data is proposed that intrinsically and structurally adjusts the test statistic for the possible presence of tied observations between the sampled populations, thereby obviating the need to require the populations to be continuous. The populations may be measurements on as low as the ordinal scale, and need not be homogeneous. In cases where the null hypotheses are rejected, the test statistic enables the determination of which of the sampled populations is likely to be responsible for the rejection (a determination which the Wilcoxon Mann Whitney test cannot handle). The proposed method is illustrated with some data, and shown to compare favorably with some existing methods available for the same purpose.

Keywords: $\quad$ Two sampled data, proposed method, observations, hypothesis.

\section{Introduction}

Suppose a researcher has collected two random samples of sizes $n_{1}$ and $n_{2}$ from two populations $x_{1}$ and $x_{2}$ respectively. This researcher may be interested in

testing a null hypothesis such as $H_{0}: \mu_{2}=\frac{\alpha}{\beta} \mu_{1}+\sigma$ verses either a two sided or any of the one sided alternative hypotheses, where $\alpha$ and $\beta$ are non-zero real numbers, and $\sigma$ is any real number including zero. The null hypothesis is that one of the populations is on the average at least (at most) a multiple (a proportion) of the other population. This situation may arise in many cases. In the health delivery system, researchers may be interested in testing the hypothesis that the effective dosage of a certain treatment drug is at least $c$ times that of a control

Dr. Ebuh is a Senior Lecturer in the Department of Statistics, Faculty of Physical Sciences. Email him at: ablegod007@yahoo.com. Professor Oyeka is Professor of Statistics in the Department of Statistics, Faculty of Physical Sciences. 


\section{EBUH \& OYEKA}

drug, where $c=\frac{\alpha}{\beta}$, or that the bed occupancy rate of public hospitals is at most $c$ times that of private hospitals. In business studies, interest may be in determining whether the cost of a certain line of products in a certain retail shop or market is at least $c$ times higher on the average than the cost in another retail shop or market, or whether Gender B workers on the average earn at most $c$ times that of their Gender A counterparts of equal skill. In education and public affairs, interest may be in whether students of a certain instructor, or candidates under a certain panel of judges, score at least $c$ times more than students of another instructor or candidates under another panel of judges; or whether the rate at which a certain set of trial judges deliver judgment in cases is at most $c$ times the rate at which a second set of trial judges deliver judgments during the year, etc.

In each of these and similar situations, the parametric $t$ test cannot properly be used to test the hypothesis without first using appropriate data transformation, given the problem of homogeneity. If $c=1$, then the $t$ test may be used to test the desired null hypothesis provided the sampled populations are mutually homogeneous and normally distributed. If $c$ is any real number other than 1 , then the $t$ test cannot be properly used for data analysis, even if the populations are normally distributed, without first applying some appropriate transformation to ensure homogeneity. This is because multiplying or dividing a data set by some non-zero constant changes the variance of the data set by the square of the constant, thereby violating the assumption of homogeneity necessary for the valid application of the $t$ test.

Rather than applying some data transformation aimed at achieving homogeneity of variances, which may not be readily available, use of nonparametric statistical methods in these situations is usually preferable. If the sampled populations are related, paired or matched, then non-parametric methods that readily suggest themselves are the sign test and the Wilcoxon's Signed Rank Sum Test (Gibbons, 1971).

The problem with these two tests is that they require the sampled populations to be continuous, thereby theoretically making no definite provisions for the possible presence of tied observations between the populations. Oyeka et al. (2009) developed a method for the analysis of these types of data that intrinsically and structurally adjusts the test statistic for the presence of any ties between the sampled populations, which may now be measurements on as low as the ordinal scale and need not be continuous. If the sampled populations are independent, then the non-parametric methods often used in their analysis include 
the median test and the Wilcoxon Mann Whitney U. Test (Gibbons, 1971; Oyeka, 2009).

A problem with these two test statistics is that they often resolve the problem of ties between sampled populations by assigning tied observations their mean ranks. If the numbers of ties are large, this approach would tend to compromise the power of the tests, which may seriously affect any conclusions based on them. An alternative non-parametric statistical method is proposed to test the desired null hypothesis when the populations are independent. The proposed method intrinsically and structurally adjusts the test statistic for the possible presence of ties between the sampled populations, obviating the need to require the populations to be continuous. These populations may therefore be measurements on as low as the ordinal scale. Other authors who have done some research in this area include Afuecheta et al (2012), Ebuh \& Oyeka (2012), \& Ebuh et al (2012).

\section{The Proposed Method}

Let $\mathrm{x}_{\mathrm{ij}}$ be the ith observation independently drawn from population $x_{\mathrm{j}}$, for $i=$ $1,2, \ldots, n_{\mathrm{j}} ; j=1,2$. Population $x_{\mathrm{j}}$ may be measurements on as low as the ordinal scale and need not be continuous. To develop the test statistic, first list unchanged all the observations $x_{\mathrm{i}}$ from one of the sampled populations $x_{1}$ while multiplying, (or dividing) each of the observations $x_{\mathrm{j} 2}$ from the other sampled population $x_{2}$ by the constant $c=\frac{\alpha}{\beta}$, then add (or subtract) the constant $d=\sigma$ before pooling them together. For the purpose of determining the common median the pooled observations are then ranked together, either from the smallest to the largest or the largest to the smallest. Tied observations are assigned their mean ranks. The common median $M c$ of the pooled sample observations is then determined. Let $r_{\mathrm{ij}}$ be the rank assigned to $x_{\mathrm{ij}}$ in the combined ranking of $n=n_{1}+n_{2}$ sample observations. The proposed method is developed based on the common median Mc in particular. For the purposes of comparison with the Wilcoxon Mann Whitney test, let $r_{\mathrm{i} 1}$ be the rank assigned $x_{\mathrm{i} 1}$ and $r_{\mathrm{j} 2}$ the rank assigned to $x_{\mathrm{j} 2}$, adjusted values of $x_{\mathrm{j} 2}$ in the combined ranking of the ' $n$ ' $=n_{1}+n_{2}$ sample observations; for $i=1,2, \ldots, n_{1}$ and $j=1,2, \ldots n_{2}$. 


\section{EBUH \& OYEKA}

Let

$$
u_{i 1}=\left\{\begin{array}{l}
1, \text { if } x_{i 1}>M c \\
0, \text { if } x_{i 1}=M c \\
-1, \text { if } x_{i 1}<M c
\end{array}\right.
$$

for $i=1,2 \ldots n_{1}$. Let

$$
\stackrel{+}{\pi_{1}}=P\left(u_{1 i}=1\right) ; \pi_{1}=P\left(u_{1 i}=0\right) ; \bar{\pi}_{1}=P\left(u_{1 i}=-1\right)
$$

where $\stackrel{+}{\pi_{1}}+\stackrel{0}{\pi_{1}}+\bar{\pi}_{1}=1$

and

$$
W_{1}=\sum_{i=1}^{n_{1}} \mu_{i 1} .
$$

$W_{1}$ is the difference between the numbers of sample observations in population $X_{1}$ that are greater than, and the number of sample observations in $X_{1}$ that are less than, the common median $M c$.

Similarly, let

$$
u_{i 2}=\left\{\begin{array}{r}
1, \text { if } x_{j 2}>M c \\
0, \text { if } x_{j 2}=M c \\
-1, \text { if } x_{j 2}<M c
\end{array}\right.
$$

for $j=1,2, \ldots, n_{2}$. Let

$$
\stackrel{+}{\pi}_{2}=P\left(u_{j 2}=1\right) ; \pi_{2}^{0}=P\left(u_{j 2}=0\right) ; \bar{\pi}_{2}=P\left(u_{j 2}=-1\right)
$$

where $\stackrel{+}{\pi_{2}}+\stackrel{0}{\pi_{2}}+\bar{\pi}_{2}=1$, and define

$$
W_{2}=\sum_{j=1}^{n_{2}} \mu_{j 2} .
$$

$W_{2}$ is the difference between the numbers of sample observations in population $X_{2}$ that are greater than, and the number of sample observations in $X_{2}$ that are less than, the common median Mc. Thus, 
$\mathrm{E}\left(\mu_{i 1}\right)=\stackrel{+}{\pi}_{1}-\bar{\pi}_{1} ; \operatorname{Var}\left(\mu_{i 1}\right)=\stackrel{+}{\pi_{1}}+\bar{\pi}_{1}-\left(\begin{array}{l}+ \\ \pi_{1}-\bar{\pi}_{1}\end{array}\right)^{2}$

and

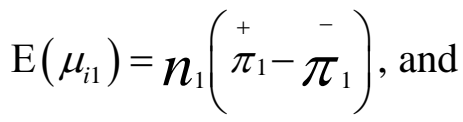

$\operatorname{Var}\left(W_{1}\right)=\sum_{i=1}^{n_{1}} \operatorname{Var}\left(\mu_{\mathrm{i} 1}\right)$. Thus,

$\operatorname{Var}\left(W_{1}\right)=n_{1}\left(\stackrel{+}{\pi}_{1}+\bar{\pi}_{1}-\left({ }^{+}-\bar{\pi}_{1}-\bar{\pi}_{1}\right)^{2}\right)$.

Note $\stackrel{+}{\pi_{1}}, \pi_{1}$ and $\bar{\pi}_{1}$ are respectively the probabilities that a randomly selected observation from population $x_{1}$ is on the average greater (higher), the same as (equal to), or smaller (lower) than the common median $M c$ of the combined sample observations. Their sample estimates are respectively

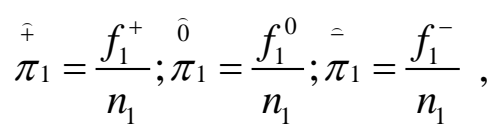

where $f_{1}^{+}, f_{1}^{0}$, and $f_{1}^{-}$are respectively the number of 1 's, 0 's and -1 's in the frequency distribution of the $\mathrm{n}_{1}$ values of these numbers in $u_{\mathrm{ij}}, \mathrm{i}=1,2, \ldots, n_{1}$.

Note from Equation 4

$$
\begin{aligned}
& \mathrm{W}_{1}=f_{1}-f_{1}^{-} \\
& \mathrm{E}\left(\mu_{i 2}\right)={ }_{\pi_{2}}^{+}-\bar{\pi}_{2} ; \operatorname{Var}\left(\mu_{\mathrm{i} 2}\right)={ }_{\pi_{2}}^{+}-\bar{\pi}_{2}-\left({ }^{+}+-\bar{\pi}_{2}\right)^{2} \\
& \mathrm{E}\left(W_{2}\right)=n_{2}\left(\stackrel{+}{ }_{2}-\bar{\pi}_{2}\right) \\
& \operatorname{Var}\left(W_{2}\right)=n_{2}\left(\stackrel{+}{\pi}_{2}+\bar{\pi}_{2}-\left(\begin{array}{l}
+ \\
\pi_{2}-\bar{\pi}_{2}
\end{array}\right)^{2}\right) .
\end{aligned}
$$

Note $\stackrel{+}{\pi}_{2}, \pi_{2}$ and $\bar{\pi}_{2}$ are respectively the probabilities that a randomly selected adjusted sample observation from population $x_{2}$ is on the average greater 


\section{EBUH \& OYEKA}

(higher), the same as (equal to), or smaller (lower) than the common median Mc. Their sample estimates are respectively

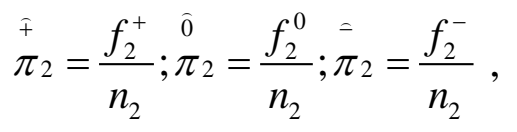

where $f_{2}^{+}, f_{2}^{0}$, and $f_{2}^{-}$are respectively the number of 1 's, 0 's and -1 's in the frequency distribution of these numbers in $u_{\mathrm{j} 2}, i=1,2, \ldots, n_{2}$.

Note from Equation 8 that

$$
W_{2}=f_{2}^{+}+f_{2}^{-}
$$

A null hypothesis that is usually of interest in two sample problems, particularly when non-parametric methods are used, is that the two populations have equal medians $M_{0}$. If population $x_{1}$ has median $M_{1}$ and population $x_{2}$ has median $M_{2}$, then a null hypothesis of interest may be

$$
H_{0}: M_{1}=M_{2}=M_{0}
$$

versus any desired alternative hypothesis.

Using the median test or the Wilcoxon Mann Whitney test to list the null hypothesis of Equation 17, and given the rejection of the null hypothesis, one could not immediately say which of the sampled populations actually led to the rejection of $H_{0}$. This is because one of the population medians may (or may not) be equal to the hypothesized value $M_{0}$ whereas the other population median may (or may not) be equal to $M_{0}$, but the test being used may not immediately reveal this pattern. To help determine this possibility, the null hypothesis of Equation 19 can be alternatively expressed as

$$
H_{01}: M_{1}=M_{0} \text { versus } H_{11}: M_{1} \neq M_{0}
$$

and

$$
H_{02}: M_{2}=M_{0} \text { versus } H_{12}: M_{2} \neq M_{0}
$$


If the null hypothesis of Equations 20 and 21 are simultaneously accepted, then the null hypothesis of Equation 19 would automatically be true. But if any of the null hypothesis of Equations 20 and 21 are rejected, then the null hypothesis of Equation 19 must also be rejected.

The null hypothesis of Equation 20 is equivalent to the null hypothesis that the proportion of all observations in population $x_{1}$ that are on the average greater (higher) than the common median of all the observations in populations $x_{1}$ and $x_{2}$ combined is equal to the proportion of all observations in population $x_{1}$ that are on the average smaller (lower) than the common median of the combined observations in populations $x_{1}$ and $x_{2}$. This is equivalent to testing the null hypothesis.

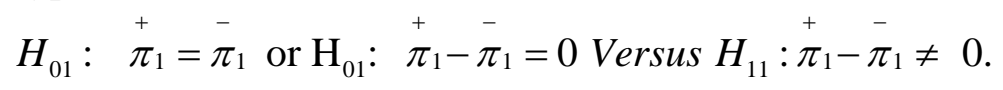

Similarly the null hypothesis of Equation 21 is equivalent to the null hypothesis.

$$
H_{02}: \stackrel{+}{\pi_{2}}=\bar{\pi}_{2} \text { or } \mathrm{H}_{02}: \stackrel{+}{\pi_{2}}-\bar{\pi}_{2}=0 \text { Versus } H_{12}: \stackrel{+}{\pi_{2}}-\bar{\pi}_{2} \neq 0 .
$$

Under the null hypothesis of equation 22, the test statistic

$$
\chi_{1}^{2}=\frac{W_{1}^{2}}{\operatorname{Var}\left(W_{1}\right)}=\frac{\left(f_{1}^{+}-f_{1}^{-}\right)^{2}}{n_{1}\left(\hat{\hat{+}} \hat{\pi_{1}}+\bar{\pi}_{1}-\left(\hat{+} \hat{\bar{t}} \bar{\pi}_{1}-\bar{\pi}_{1}\right)^{2}\right)}
$$

under $H_{01}$ has approximately the chi-square distribution with 1 degree of freedom. Similarly, under the null hypothesis of Equation 23 the test statistic

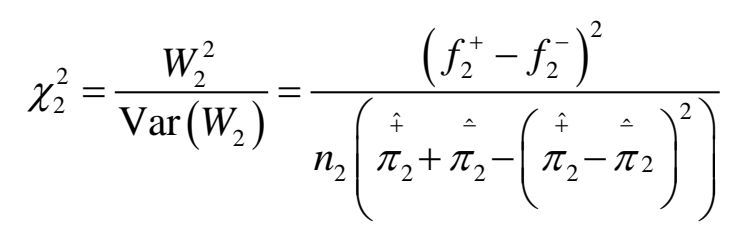

has approximately the chi-square distribution with 1 degree of freedom.

The null hypothesis of Equations 22 and 23 are each rejected if the calculated chi-square values are at least equal to the tabulated or critical chisquare value with 1 degree of freedom for a specified $\alpha$ level; otherwise the null hypothesis is accepted. 


\section{EBUH \& OYEKA}

Finally, the proposed method may be easily modified and used to test a hypothesis concerning appropriately chosen measures of central tendency for two populations when $c=\frac{\alpha}{\sqrt{3}}=1$, and $d=\alpha=0$.

\section{Illustrative Example}

Suppose Gender B students on the average earned one grade lower than their Gender A colleagues. On the basis of this finding, the instructor required Gender B students to mandatorily attend tutorials. The question arose whether the instructor was justified in this policy. Data were collected on a random sample of Gender A and Gender B students.

\section{Gender \\ $\begin{array}{llllllllllllllll}\text { A } & \mathrm{B}^{+} & \mathrm{A} & \mathrm{A}^{-} & \mathrm{A} & \mathrm{C}^{+} & \mathrm{A}^{-} & \mathrm{A}^{+} & \mathrm{A}^{+} & \mathrm{C}^{+} & \mathrm{A}^{+} & \mathrm{C} & \mathrm{A}^{+} & \mathrm{A}^{-} & \mathrm{A}^{+}\end{array}$ \\ Students \\ Gender \\ $\begin{array}{lllllllllllllllllllll} & \text { B } & \mathrm{B}^{+} & \mathrm{F} & \mathrm{F} & \mathrm{B} & \mathrm{A}^{-} & \mathrm{D} & \mathrm{B}^{+} & \mathrm{C}^{+} & \mathrm{B} & \mathrm{B}^{-} & \mathrm{A}^{+} & \mathrm{B} & \mathrm{F} & \mathrm{E} & \mathrm{C}^{+} & \mathrm{A}^{+} & \mathrm{C}^{-} & \mathrm{E} & \mathrm{C}^{-}\end{array}$ Students}

First, list the Gender A students' letter grades unchanged, here designated as $\mathrm{x}_{\mathrm{i} 1}$, and then list the Gender B students' grades after increasing each of them by one grade level, here designated as $x_{\mathrm{j} 2}$; the resulting grades are then pooled together and ranked from the highest, assigned the rank 1, to the lowest, assigned the rank 33. Tied grades are as usual assigned their mean ranks. The common median grade of the pooled sample is found to be a $\mathrm{B}^{+}$.

Equations 1 and 5 are now applied to the listed data. The values of $u_{\mathrm{i} 1}, u_{\mathrm{j} 2}$ and the corresponding ranks are presented in Table 1 . From the values of $u_{i 1}$ in column 6 of Table 1 it is shown that

$$
\begin{gathered}
f_{1}^{+}=10, f_{1}^{0}=1 \text { and } f_{1}^{-}=3 \text { so that } \\
\stackrel{\uparrow}{\pi_{1}}=\frac{10}{14}=0.714, \stackrel{\hat{0}}{\pi_{1}}=\frac{1}{14}=0.071 \text { and } \stackrel{\hat{\pi}}{\pi_{1}}=\frac{3}{14}=0.214
\end{gathered}
$$

Also from Equation 11, it is found that the estimated variance of $W_{1}$ is 
NON-PARAMETRIC METHOD FOR ANALYSIS OF TWO SAMPLED DATA

Table 1: Values of $u_{i 1}, u_{\mathrm{j} 2}$ and other Statistics for the illustrative Example

\begin{tabular}{|c|c|c|c|c|c|c|}
\hline \multirow[b]{2}{*}{$\begin{array}{l}\text { Gender A } \\
\text { Grade } x_{i 1}\end{array}$} & \multirow[b]{2}{*}{$\begin{array}{l}\text { Gender B } \\
\text { Grade } x_{\mathrm{j} 2}\end{array}$} & \multirow[b]{2}{*}{$\begin{array}{l}\text { Adjusted } \\
\text { Gender B } \\
\text { Grade } x_{j 2}\end{array}$} & \multicolumn{2}{|c|}{ Rank of Ranking } & \multirow{2}{*}{$\begin{array}{l}u_{\mathrm{i} 1} \\
\text { (Eqn1) }\end{array}$} & \multirow{2}{*}{$\begin{array}{l}u_{\mathrm{i} 2} \\
(\text { Eqn5) }\end{array}$} \\
\hline & & & $\begin{array}{l}x_{i 1} \text { in the } \\
\text { combined } \\
\text { ranking }\left(n_{\mathrm{i} 1}\right)\end{array}$ & $\begin{array}{l}x_{\mathrm{j} 2} \text { in the } \\
\text { combined } \\
\text { ranking }\left(n_{\mathrm{i} 1}\right)\end{array}$ & & \\
\hline $\mathrm{B}^{+}$ & $\mathrm{B}^{+}$ & $A^{-}$ & 17.5 & 13 & 0 & 1 \\
\hline$A$ & $\mathrm{~F}$ & $E$ & 9 & 32 & 1 & -1 \\
\hline$A^{-}$ & $\mathrm{F}$ & $E$ & 13 & 32 & 1 & -1 \\
\hline $\bar{A}$ & $\mathrm{~B}$ & $\mathrm{~B}^{+}$ & 9 & 17.5 & 1 & 1 \\
\hline $\mathrm{C}^{+}$ & $A^{-}$ & A & 23.5 & 9 & -1 & 1 \\
\hline$A^{-}$ & $\mathrm{D}$ & $\mathrm{C}^{-}$ & 13 & 28 & 1 & -1 \\
\hline$A^{+}$ & $\mathrm{B}^{+}$ & $A^{-}$ & 4 & 13 & 1 & 1 \\
\hline$A^{+}$ & $\mathrm{C}^{+}$ & $\mathrm{B}^{-}$ & 4 & 21.5 & 1 & 0 \\
\hline $\mathrm{C}^{+}$ & B & $\mathrm{B}^{+}$ & 23.5 & 17.5 & -1 & 1 \\
\hline$\overline{A^{+}}$ & $\mathrm{B}^{-}$ & $\mathrm{B}$ & 4 & 20 & 1 & 1 \\
\hline $\mathrm{C}$ & $A^{+}$ & $A^{+}$ & 26 & 4 & -1 & 1 \\
\hline$A^{+}$ & $B$ & $\mathrm{~B}^{+}$ & 4 & 17.5 & 1 & 1 \\
\hline$A^{-}$ & $\mathrm{F}$ & $E$ & 13 & 32 & 1 & -1 \\
\hline \multirow[t]{6}{*}{$A^{+}$} & $E$ & $\mathrm{D}$ & 4 & 29.5 & 1 & -1 \\
\hline & $\mathrm{C}^{+}$ & $\mathrm{B}^{-}$ & & 21.5 & & 0 \\
\hline & $A^{+}$ & $A^{+}$ & & 4 & & 1 \\
\hline & $\mathrm{C}^{-}$ & $C$ & & 26 & & -1 \\
\hline & $E$ & $\mathrm{D}$ & & 29.5 & & -1 \\
\hline & $\mathrm{C}^{-}$ & C & & 26 & & -1 \\
\hline Total & & & 167.5 & 393.5 & & \\
\hline
\end{tabular}

$$
\begin{array}{lll}
\operatorname{Var}\left(W_{1}\right) \quad & =14\left((0.714+0.214)-(0.714-0.214)^{2}\right) \\
& =\quad 14(0.928-0.25)=14(0.678)=9.492
\end{array}
$$

To test the null hypothesis of Equation 22 that Gender A s have the same median grades as the entire class (both genders combined), Equation 24 shows that 


\section{EBUH \& OYEKA}

$$
\chi_{1}^{2}=\frac{(7)^{2}}{9.492}=\frac{49}{9.492}=5.162 \quad(p \text { value }=0.0231)
$$

which with 1 degree of freedom is statistically significant, leading to a rejection of the null hypothesis. From the values of $u_{\mathrm{j} 2}$ in column 7 of Table 1

$$
\begin{aligned}
& f_{2}^{+}=9, f_{2}^{0}=2 \text { and } f_{2}^{-}=8 \text { so that } \\
& {\stackrel{\bar{f}}{\pi_{2}}}_{2}=\frac{9}{19}=0.474, \hat{\pi}_{2}=\frac{2}{19}=0.105 \text { and } \hat{\bar{\pi}}=\frac{8}{19}=0.421 \text {. }
\end{aligned}
$$

From Equation 8, $W_{2}=f_{2}^{+}-f_{2}^{-}=9-8=1$. The estimated variance of $W_{2}$ is from Equation 15

$$
\begin{array}{lll}
\operatorname{Var}\left(W_{2}\right) \quad & =19\left((0.474+0.421)-(0.474-0.421)^{2}\right) \\
& = & 19(0.895-0.003)=19(0.892)=16.948
\end{array}
$$

The test statistic for the null hypothesis of Equation 23 that Gender B students have the same median score as the overall class in the course is from Equation 25.

$$
\chi_{2}^{2}=\frac{(1)^{2}}{16.948}=0.059 \quad(p \text { value }=0.8081)
$$

which with 1 degree of freedom is not statistically significant, leading to an acceptance of the null hypothesis of Equation 23. Because the null hypothesis of Equations 22 and 23 are not both accepted, the null hypothesis of Equation 19 cannot be accepted. It can therefore be concluded on the basis of these tests that the hypothesized relationship between Gender A and Gender B performances in the course may not be valid.

Note that the median grade in the course for Gender A students is about an A whereas the unadjusted or original median grade for Gender B students is about a $\mathrm{C}^{+}$in the fabricated example. Hence, if the hypothesized relative relationship between Gender A and Gender B student grades were to hold then the original median grade for Gender B students would be expected to be about an A', which the adjusted Gender B median grade does not attain.

If the Wilcoxon Mann Whitney test had been used to analyze the data, it could be shown with $R_{1}=167.5$ (see Table 1 ) that 


$$
\begin{aligned}
& U=n_{1} n_{2}+\frac{n_{1}\left(n_{1}+1\right)}{2}-R_{1}=(14)(19)+\frac{14(15)}{2}-167.5 \\
& =266+105-167.5=203.5 \text { with mean } \mathrm{E}(U)=\frac{n_{1} n_{2}}{2}=\frac{(14)(19)}{2}=133
\end{aligned}
$$

and

$$
\operatorname{Var}(u)=\frac{n_{1} n_{2}\left(n_{1}+n_{2}+1\right)}{12}=\frac{(14)(19)(34)}{12}=753.667 .
$$

Thus, $\operatorname{Se}(u)=\sqrt{753.667}=27.453$.

The test statistic for the null hypothesis of Equation 19 for the equality of the two population medians is

$$
Z=\frac{u-E(u)}{S e(u)}=\frac{203.5-133}{27.453}=\frac{70.5}{27.453}=2.568 \quad(\mathrm{p} \text { value }=0.0102)
$$

which is statistically significant with nominal alpha set to 0.05 .

The Wilcoxon Mann Whitney test, like the proposed test statistic, retained as tenable the null hypothesis of Equation 19. However, use of the usual median test with the data yielded a Chi-squared value of 0.24 , which was not statistically significant, and led to an acceptance of the null hypothesis of equal population medians. This is probably due to the occasional inability of the usual median test to adequately provide for the presence of ties between the sampled populations, which may lead to an acceptance of a false null hypothesis.

From the values of $u_{\mathrm{i} 1}$ in column 6 of the Table 2 it is shown that

$$
\begin{gathered}
f_{1}^{+}=10, f_{1}^{0}=1 \text { and } f_{1}^{-}=3 \text { so that } \\
\stackrel{+}{\pi_{1}}=\frac{10}{14}=0.714, \stackrel{\hat{0}}{1}_{1}=\frac{1}{14}=0.071 \text { and } \hat{\pi_{1}}=\frac{3}{14}=0.214 .
\end{gathered}
$$

From Equation $4 W_{1}=f_{1}^{+}-f_{1}^{-}=10-3=7$. From Equation 11 it is shown that the estimated variance of $W_{1}$ is

$$
\begin{array}{rll}
\operatorname{Var}\left(W_{1}\right) \quad & =14\left((0.714+0.214)-(0.714-0.214)^{2}\right) \\
& = & 14(0.928-0.25)=14(0.678)=9.492 .
\end{array}
$$




\section{EBUH \& OYEKA}

Table 2: Values of $u_{\mathrm{i} 1}, u_{\mathrm{j} 2}$ and other Statistics for simulated data

\begin{tabular}{|c|c|c|c|c|c|c|}
\hline \multirow[b]{2}{*}{$\begin{array}{l}\text { Gender A } \\
\text { Grade } x_{i 1}\end{array}$} & \multirow[b]{2}{*}{$\begin{array}{l}\text { Gender B } \\
\text { Grade } x_{j 2}\end{array}$} & \multirow{2}{*}{$\begin{array}{l}\text { Adjusted } \\
\text { Gender B } \\
\text { Grade } x_{\mathrm{j} 2}\end{array}$} & \multicolumn{2}{|c|}{ Rank of Ranking } & \multirow{2}{*}{$\begin{array}{l}u_{\mathrm{i} 1} \\
\text { (Eqn1) }\end{array}$} & \multirow{2}{*}{$\begin{array}{l}u_{\mathrm{i} 2} \\
\text { (Eqn5) }\end{array}$} \\
\hline & & & $\begin{array}{l}x_{i 1} \text { in the } \\
\text { combined } \\
\text { ranking }\left(n_{i 1}\right)\end{array}$ & $\begin{array}{l}x_{\mathrm{j} 2} \text { in the } \\
\text { combined } \\
\text { ranking }\left(\mathrm{n}_{\mathrm{i} 1}\right)\end{array}$ & & \\
\hline $\mathrm{B}+$ & $\mathrm{C}-$ & $\mathrm{B}+$ & 15 & 26.5 & 1 & -1 \\
\hline A & $\mathrm{C}+$ & $E$ & 8.5 & 22.5 & 1 & 0 \\
\hline $\mathrm{A}+$ & $E$ & A- & 4 & 29.5 & 1 & -1 \\
\hline A- & $\mathrm{B}+$ & $\mathrm{C}$ & 11.5 & 15 & 1 & 1 \\
\hline C & $\mathrm{B}+$ & $A+$ & 25 & 15 & -1 & 1 \\
\hline $\mathrm{A}+$ & $D$ & B- & 4 & 28 & 1 & -1 \\
\hline$A+$ & $\mathrm{F}$ & $\mathrm{B}+$ & 4 & 32 & 1 & -1 \\
\hline A- & A- & $\mathrm{A}+$ & 11.5 & 11.5 & 0 & 1 \\
\hline$A$ & $\mathrm{C}+$ & $A$ & 8.5 & 22.5 & 1 & 0 \\
\hline A- & $B$ & $D$ & 11.5 & 18 & 1 & 1 \\
\hline $\mathrm{C}+$ & B- & B- & 22.5 & 20 & -1 & 1 \\
\hline$A+$ & $E$ & $A+$ & 4 & 29.5 & 1 & -1 \\
\hline $\mathrm{C}+$ & B & C & 22.5 & 18 & -1 & 1 \\
\hline \multirow[t]{6}{*}{$A+$} & $\mathrm{F}$ & $\mathrm{B}+$ & 4 & 32 & 1 & -1 \\
\hline & $\mathrm{B}$ & $B$ & & 18 & & 1 \\
\hline & $A+$ & $\mathrm{D}$ & & 4 & & 1 \\
\hline & $A+$ & $E$ & & 4 & & 1 \\
\hline & C- & C- & & 26.5 & & -1 \\
\hline & $F$ & $E$ & & 32 & & -1 \\
\hline Total & & & 156.5 & 404.5 & & \\
\hline
\end{tabular}

To test the null hypothesis of Equation 22 that Gender A students have the same median grades as the entire class (both genders combined) it can be shown from Equation 24 that

$$
\chi_{1}^{2}=\frac{(7)^{2}}{9.492}=\frac{49}{9.492}=5.162 \quad(p \text { value }=0.0231) \text {, }
$$

which with 1 degree of freedom is statistically significant, leading to a rejection of the null hypothesis.

Furthermore, from the values of $u_{\mathrm{j} 2}$ in column 7 of Table 2 it is shown that 
$f_{2}^{+}=9, f_{2}^{0}=2$ and $f_{2}^{-}=8$ so that

${\stackrel{\mp}{\pi_{2}}}_{2}=\frac{9}{19}=0.474, \hat{0}_{2}=\frac{2}{19}=0.105$ and $\hat{\bar{\pi}}_{2}=\frac{8}{19}=0.421$.

From Equation $8 W_{2}=f_{2}^{+}-f_{2}^{-}=9-8=1$. The estimated variance of $W_{2}$ from Equation 15 is

$$
\begin{array}{rll}
\operatorname{Var}\left(W_{2}\right) \quad & =19\left((0.474+0.421)-(0.474-0.421)^{2}\right) \\
& =19(0.895-0.003)=19(0.892)=16.948 .
\end{array}
$$

The test statistic for the null hypothesis of Equation 23 that Gender B students have the same median score as the overall class in the course is from Equation 25.

$$
\chi_{2}^{2}=\frac{(1)^{2}}{16.948}=0.059 \quad(\mathrm{p} \text { value }=0.8081)
$$

which with 1 degree of freedom is not statistically significant, leading to an acceptance of the null hypothesis of Equation 23.

Because the null hypothesis of Equations 22 and 23 are not both accepted, the null hypothesis of Equation 19 cannot be accepted. It can therefore be concluded on the basis of these tests that the hypothesized relationship between Gender A and Gender B performances in the course may not be valid.

Note that the median grade in the course for Gender A students is about an A whereas the unadjusted or original median grade for Gender B students is about a $\mathrm{C}^{+}$. Hence if the hypothesized relative relationship between Gender A and Gender B student grades were to hold, then the original median grade for Gender B students would be expected to be about an A', which the adjusted Gender B median grade does not attain.

Using the Wilcoxon Mann Whitney $\mathrm{U}$ test to analyze the data would have the result, with $R_{1}=156.5$ (see Table 2), that

$$
\begin{aligned}
U & =n_{1} n_{2}+\frac{n_{1}\left(n_{1}+1\right)}{2}-R_{1}=(14)(19)+\frac{14(15)}{2}-156.5 \\
& =266+105-156.5=214.5 \text { with mean } \mathrm{E}(U)=\frac{n_{1} n_{2}}{2}=\frac{(14)(19)}{2}=133
\end{aligned}
$$




\section{EBUH \& OYEKA}

and variance.

$$
\operatorname{Var}(u)=\frac{n_{1} n_{2}\left(n_{1}+n_{2}+1\right)}{12}=\frac{(14)(19)(34)}{12}=753.667
$$

Hence, $\operatorname{Se}(u)=\sqrt{753.667}=27.453$.

The test statistic for the null hypothesis of Equation 19 for the equality of the two population medians is

$$
Z=\frac{u-E(u)}{S e(u)}=\frac{214.5-133}{27.453}=\frac{81.5}{27.453}=2.969 \quad(\mathrm{p} \text { value }=0.0030)
$$

which is statistically significant, and the discussion on the previous example is repeated.

\section{Conclusion}

A non-parametric statistical method for the analysis of two sample data was presented that may be applied on measurements on as low as the ordinal scale and need not be homogeneous. The test statistic is intrinsically and structurally adjusted to provide for the possibility of any tied observations between the sampled populations and hence obviates the need to require the populations to be continuous. When the null hypothesis is rejected, it indicates which of the sampled populations may have been responsible for the rejection (a determination which the Wilcoxon Mann Whitney test cannot handle). Results from an example suggest that the test statistic may be as powerful as the Wilcoxon Mann Whitney test, and more powerful than the usual median test.

\section{References}

Afuecheta, E. O., Oyeka, I. C. A, Ebuh, G. U., \& Nnanatu, C. C. (2012). Modified Median Test Intrinsically Adjusted For Ties. Journal of Basic Physical Research, 3, 30-34.

Ebuh, G. U. \& Oyeka, I. C. A. (2012). Statistical Comparison of Eight Alternative Methods for the Analysis of Paired Sample Data with Applications. Open Journal of Statistics (OJS), 2(3), 328-345. 
Ebuh, G. U., Oyeka, I. C. A., \& Nwosu, C. R. (2012). Application of Dummy Variables Multiple Regression to Paired Samples with three Options. Journal of Nigerian Statistical Association (JNSA) 24, 34-44

Gibbons, J. D. (1971). Non-parametric statistical inference. New York: McGraw Hill.

Oyeka, I. C. A., Ebuh, G. U., Nwosu, C. R., Utazi, E. C., Ikpegbu, P. A., Obiora-Ilouno, H., \& Nwankwo, C. C. (2009). A Method of Analysing Paired Data Intrinsically Adjusted for Ties. Global Journal of Mathematics and Statistics, India. 1(1), 1-6

Oyeka, I. C. A. (2009). An Introduction to applied statistical methods, 8th edition. Enugu: Nobern Avocation Publishing Company, 457-495. 